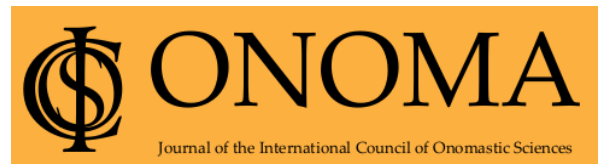

Onoma 56

Journal of the International Council of Onomastic Sciences

ISSN: 0078-463X; e-ISSN: 1783-1644

Journal homepage: https://onomajournal.org/

\title{
Changements anthroponymiques et migration : la commune de Certeze, au nord-ouest de la Roumanie
}

\section{Adelina Emilia Mihali*}

L'Institut de Linguistique et d'Histoire Littéraire « Sextil Pușcariu » de L'Académie Roumaine, Filiale de Cluj-Napoca

To cite this article: Mihali, Adelina Emilia. 2021. Changements anthroponymiques et migration : la commune de Certeze, au nord-ouest de la Roumanie. Onoma 56, 87-106. DOI: 10.34158/ONOMA.56/2021/5

To link to this article: https://doi.org/10.34158/ONOMA.56/2021/5

(C) Onoma and the author.

\section{Article history}

Received on 25 July 2021.

Final form accepted on 29 November 2021.

Published online on 13 December 2021.

Anthroponymic changes and migration: The case of Certeze village, northwest Romania

Abstract: This paper is a sociolinguistic analysis of the anthroponymic changes in northwest Romania, Certeze commune, Satu Mare county, from where a large part of the population has emigrated, definitively or temporarily. The article follows the impact of migration in the innovation of first names and aims at deciphering the sociolinguistic mechanisms that underlie the choice of names. The period under investigation includes the post-revolutionary age and the beginning of migration, the increase in the rate of migration, the economic crisis, and the remigration of emigrants.

* Contact: L'Institut de Linguistique et d'Histoire Littéraire "Sextil Pușcariu » de L'Académie Roumaine, Filiale de Cluj-Napoca, 21 rue Emil Racoviţă, 400165, ClujNapoca, Roumanie, adelinatatar@yahoo.com 
The study tries to provide answers to questions regarding 1) the dynamic of anthroponymic preferences in the period between the years 2000 and 2020 in Certeze, 2 ) the predominant types of names, according to structure, origin and semantics, and 3) the motivations behind the names in Romania and in the diaspora.

Keywords: Anthroponymy, migration, identity.

\section{Changements anthroponymiques et migration : la commune de Certeze, au nord-ouest de la Roumanie}

Résumé : Cette étude représente une analyse sociolinguistique de la dynamique anthroponymique de la commune de Certeze, au département de Satu Mare, où une grande partie de la population a émigré, définitivement ou temporairement, poursuivant l'influence de la migration sur l'innovation des noms de baptême et ainsi que sur l'explication des mécanismes sociolinguistiques trouvés à l'origine du choix des noms. La période étudiée comprend l'ère postrévolutionnaire et le début de la migration, l'augmentation du taux migratoire, la crise économique et la « remigration » des émigrants éventuels. L'étude se poursuit par des réponses à des questions concernant : 1) la dynamique des préférences anthroponymiques pendant la période 2000-2020 à Certeze, 2) les types de noms prédominants, en fonction de leur structure, de leur origine et de la sémantique et 3 ) les motivations trouvées derrière les noms roumains et ceux de la diaspora roumaine.

Mots-clés : Anthroponymie, migration, identité.

\section{Anthroponymische Veränderungen und Migration: Gemeinde Certeze im Nordwesten Rumäniens}

Zusammenfassung: Dieses Studium ist eine soziolinguistische Analyse anthroponymischer Veränderungen im Nordwesten Rumänien, Gemeinde Certeze, Landkreis Satu Mare, wo ein großer Teil der Bevölkerung endgültig oder vorübergehend ausgewandert ist, nachdem die Migration die Innovation von Taufnamen beeinflusst und die soziolinguistischen Mechanismen entschlüsselt hat, die der Wahl der Namen zugrunde liegen. Das Korpus des Papiers besteht aus den Namen der zwischen 2000 und 2020 geborenen Kinder, die aus den Zivilregistern entnommen wurden. Der Untersuchungszeitraum umfasst die postrevolutionäre Ära und den Beginn der Migration, den Anstieg der Migrationsrate, die Wirtschaftskrise und die Rückwanderung von Auswanderern. Die Studie versucht, Fragen zu 1) der Dynamik anthroponymischer Präferenzen in den Jahren 2000-2020 in Certeze, 2) den vorherrschenden Namenstypen je nach Struktur, Herkunft und Semantik und 3) den Motivationen für Namen in Rumänien und der Diaspora zu beantworten.

Schlüsselbegriffe: Anthroponymie, Migration, Identität. 


\title{
Changements anthroponymiques et migration : la commune de Certeze, au nord-ouest de la Roumanie
}

\author{
AdELINA EMiLia MiHALi
}

\section{Introduction}

Le nom propre, comme objet d'étude de l'onomastique, se différencie du nom commun par sa fonction spécifique, la dénomination par l'individualisation. En tant que signe linguistique, le nom propre se comporte différemment, supposant des problèmes de nature grammaticale et sémantique. De même, il peut être considéré tel un signe social, un indicateur des changements à l'intérieur d'une communauté. La raison du choix d'un nom est étroitement liée à des aspects extralinguistiques : le mouvement de la population, le statut d'une certaine langue dans une communauté, les politiques dénominatives au niveau macrosocial, les normes sociales et les phénomènes de mode ou de distinction sociale, le degré culturel ou d'émancipation des personnes qui choisissent les noms, l'intelligence, le désir, la passion des parents comme individus nommant (Fernández Juncal 2018 : 85-86 ; Troplini 2015 : 377). Les changements survenus dans la société, pendant une certaine période, influencent en grande partie la structure et la pratique de la dénomination dans cette même communauté. Par conséquent, l'étude diachronique et synchronique des noms de personne peut mettre en évidence les variations territoriales de certains anthroponymes, leur dynamique, les coutumes liées au choix du nom, c'est-àdire les principes trouvés à la base de la dénomination personnelle.

En Roumanie, l'ouverture des frontières après 1990, à la suite de la chute du régime politique communiste et l'intensification de la migration après 2000, surtout celle de l'émigration, dont l'effet au niveau anthroponymique constitue l'objet de la présente étude, ont conduit à des changements socioculturels importants, favorisant la connaissance d'autres cultures et l'accentuation de la relation entre l'identité et l'altérité. Selon les données offertes par 1'ONU1', en 2015, la Roumanie avait plus de 3, 4 millions d'émigrants, desquels plus de 2, 9 millions étaient partis pour l'Europe.

Le contact avec l'Occident a été ressenti dans les relations interpersonnelles, dans le rapport avec la tradition et avec la localité d'origine, etc. Cependant,

Les données ont été recueillies de https://www.un.org/development/desa/pd/content/ international-migrant-stock (page consultée en mai 2019). 
ces migrants n'ont pas définitivement rompu leurs relations avec la Roumanie, choisissant d'investir leur argent gagné à l'étranger dans leur pays natal, où ils se sont construits de grandes maisons à la place des maisons traditionnelles. Par l'acquisition de voitures chères, la construction de maisons imposantes et par le niveau de vie élevé exposé pendant les courtes périodes de temps passées en Roumanie, les émigrants veulent imposer dans la communauté d'origine une certaine image. Ces dernières années, à cause de la situation économique européenne, une partie de ces émigrants sont revenus dans leur pays d'origine. Dans ce contexte, les choix anthroponymiques faits par les parents, surtout par ceux partis à l'étranger, doivent être liés à leurs aspirations socioculturelles et identitaires.

\section{Le but de l'étude et la méthodologie}

Notre étude constitue une analyse sociolinguistique de la dynamique anthroponymique de la commune de Certeze, du Pays de Oaş, au département de Satu Mare. La commune de Certeze, formée des villages Certeze (chef-lieu de la commune), Huta-Certeze et Moișeni, est située au nord-ouest de la Roumanie, dans le département de Satu Mare, à $25 \mathrm{~km}$ de la frontière avec l'Ukraine (Figure 1). Les dates d'attestation de ces trois localités diffèrent : d'après Suciu (1967 : 134), la localité de Certeze est attestée dès 1490, sous le nom d'Awas ${ }^{2}$, celle de Huta-Certeze, en 1909 - Huta Certeze, magh. Lajosvölgyi huta, hameau du village de Certeze (Suciu 1967 : 299), et, celle de Moișeni, en 1828 - Mózesfalu, Mosény (Suciu 1967 : 405).

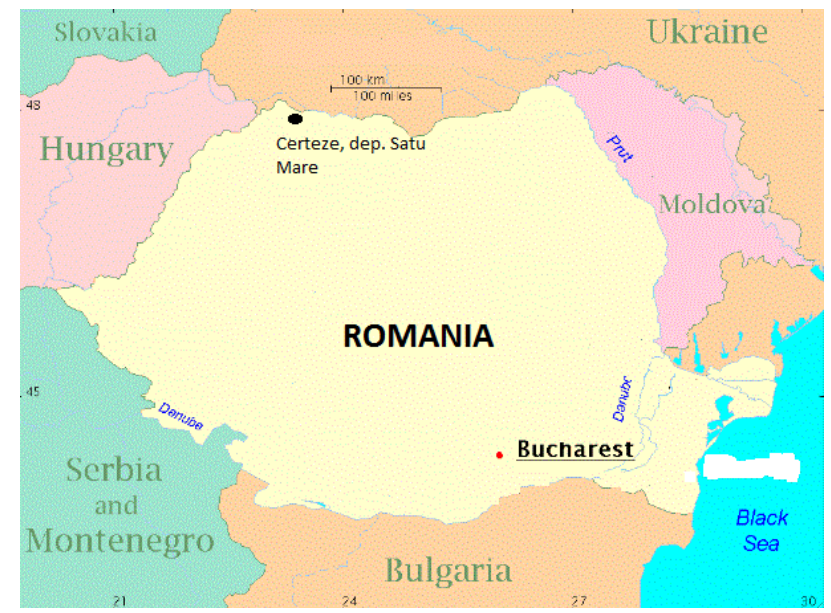

Figure 1 : Localisation de la commune sur la carte de la Roumanie ${ }^{3}$

2 D'autres attestations : 1493 Vyfalw, Wyfalw, 1828 Avass Úifalu (Suciu 1967 : 134).

3 La carte a été extraite de https://www.qsl.net/yo5ofh/common_pictures/romania.jpg (page consultée en mai 2019) et modifiée par l'auteur. 
Conformément au recensement de l'année 2011, la commune avait une population stable de 5636 habitants (3171 à Certeze, 1391 à Moișeni et 1074 à Huta-Certeze $)^{4}$. Du point de vue ethnique, la majorité est représentée par les Roumains $(93,97 \%)$, à côté desquels se trouve une minorité de Slovaques $(1,58 \%)$, surtout dans le village de Huta-Certeze.

Dans le département de Satu Mare, en 2011, selon de dernier recensement de la population, 12. 746 personnes étaient établies depuis plus d'un à l'étranger (4.475 en Italie, 1402 au Portugal, 1224 en France, 941 en Hongrie, 911 en Espagne, 698 en Grande-Bretagne, 664 en Autriche, 663 en Belgique, 608 en Allemagne, etc.) et 17.276 personnes étaient parties pour une certaine période à l'étranger (la plupart, 5170 personnes, en France et 5042 personnes en Italie) ${ }^{5}$. Dans une étude de l'année 1996 sur les départs des habitants du Pays de Oaș à l'étranger, on soutient qu'environ $60 \%$ des migrants arrivés en France proviennent de Certeze (Diminescu 1996 : 22). À présent, la mairie de Certeze nous a fourni un nombre de 480 migrants, auquel on rajoute aussi les migrants saisonniers. De même, il faut aussi tenir compte du fait que, selon les données offertes par l'Institut National de la Statistique, pendant la période 1994-2019, seulement 233 habitants de Certeze ont émigré définitivement, en changeant de domicile ${ }^{6}$.

La migration vers l'étranger a déterminé des changements socioculturels, dans cette commune parmi lesquels plusieurs que l'on peut qualifier d'anthroponymiques. L'acculturation est évidente en particulier dans l'architecture. Les émigrants de Certeze, qui ont investi leur argent en Roumanie, ont construit d'immenses villas, qui sont restées désertes pendant l'année, à l'exception des mois d'août et de décembre, quand les émigrants reviennent en vacances en Roumanie pour organiser des noces (avec des milliers de participants) et pour célébrer Noël.

Nous pouvons, maintenant, observer l'influence de l'émigration sur l'innovation des noms de baptême enregistrés en Roumanie et l'explication des mécanismes sociolinguistiques trouvés à l'origine du choix des noms. Le corpus de l'étude est constitué par les prénoms donnés aux nouveau-nés pendant la période 2000-2020, extraits du registre des transcriptions des naissances enregistrées à l'étranger ${ }^{7}$, complétées par les noms des enfants inscrits à l'école communale. Les documents consultés contiennent également des données concernant les noms des parents, une recherche qui a permis de réaliser une

4 Les données ont été recueillies de http://www.recensamantromania.ro/rezultate-2/ (page consultée en mai 2019).

5 Les données ont été recueillies de http://www.recensamantromania.ro/rezultate-2/ (page consultée en mai 2019).

$6 \mathrm{http} / /$ statistici.insse.ro:8077/tempo-online/\#/pages/tables/insse-table (page consultée en mai 2019).

7 Le citoyen roumain est obligé qu'en terme de six mois dès la naissance de son enfant demande la transcription du certificat/ extrait de naissance à la Mairie de domicile de l'un des parents. 
analyse diachronique de l'anthroponymie de la région. Afin de pouvoir suivre les motivations trouvées derrière les noms, lorsque cela a été possible, des discussions libres avec les parents ou avec des membres de la famille ont été organisées. Enfin, cette partie de l'étude est complétée par des réponses à plusieurs questions concernant : 1) la dynamique des préférences dans le choix des prénoms pendant la période 2000-2020, à Certeze, 2) les types de noms prédominants, en fonction de leur structure, de l'origine et de la sémantique et 3) les motivations trouvées derrière les noms venant de Roumanie et ceux de la diaspora.

Pendant la période 2000-2020, dans le registre des transcriptions des certificats de naissance $(90 \%$ représentant des transcriptions des certificats de naissance choisis à l'étranger et seulement $10 \%$ des duplicata) sont enregistrées 414 personnes. Dans les listes reçues de l'école de la localité apparaissent 61 élèves nés entre les années 2006-2014. Les 475 noms enregistrés (206 filles, 249 garçons) ont été groupés en fonction de deux périodes de temps, tenant compte du flux migratoire: 2000-2010, respectivement 2011-2020. Pendant la première période, ont été enregistrés 184 nouveau-nés ( 86 filles, 98 garçons) et 271 (120 filles, 151 garçons) pendant la deuxième période.

\section{Le choix du nom}

\subsection{Le nom et l'identité}

Soit qu'il s'agisse d'émigrants, d'immigrants ou d'indigènes, les parents ont la tendance de choisir pour leur enfant un nom considéré important pour l'évolution ultérieure de celui-ci, projetant sur l'enfant certaines de leurs attentes sociales (voir aussi Aldrin 2014, 2017 ; Bucholtz 2003 ; Watzlawik et al. 2016). Dans le contexte de la migration, des décisions anthroponymiques différentes peuvent conduire à la naissance d'identités ethniques distinctes pour l'enfant (Aldrin 2009 : 88). L'enfant peut assumer l'identité imposée par le nom donné par ses parents ou il peut se créer une autre identité, en fonction du milieu de vie, de la culture, des relations sociales, etc. L'étude de la dynamique de l'anthroponymie d'un pays fournit donc des données importantes sur la manière dans laquelle les citoyens se voient comme représentants d'un peuple, d'une famille, comme individus, mais aussi de la manière dont ils perçoivent et acceptent les autres. De même, l'acte de dénomination reflète la modalité à travers laquelle les gens utilisent le langage pour exprimer leur culture, leur foi, leur perception de l'environnement (Abubakari $2020: 23$ ).

L'acte de dénomination peut être ainsi analysé tant au niveau individuel qu'au niveau social, étant donc influencé par des aspects socioculturels. Dans les communautés qui respectent la tradition de la transmission d'un nom des parents/grands parents au fils/ neveu, il devient une liaison entre les générations, la différenciation étant réalisée par l'option pour un hypocoristique ou un diminutif ou pour un prénom composé. 
Le nom devient un indice des interactions socioculturelles des individus, des changements de perception à la suite du contact avec l'altérité, mais aussi des attentes des nommeurs, qui choisissent un nom pour le futur. La décision des parents à l'égard du nom choisi pour leur nouveau-né est influencée par le désir de se différencier du reste de la communauté, mais aussi par celui de s'intégrer dans un groupe, aspect évident dans la société contemporaine, quand l'anthroponymie est soumise à un processus d'internationalisation comme conséquence de la migration et de la globalisation. Le nom a donc une fonction unificatrice, réunissant les porteurs de certains noms de baptême dans une famille ou dans une collectivité, une fonction de différenciation et une fonction de prestige, car les personnes situées sur une position inférieure dans la communauté imiteront des modèles, pendant que celles trouvées au sommet de la hiérarchie sociale essaieront de se différencier des masses par les prénoms choisis (Felecan O. 2013 : 93-94).

L'étude diachronique de la transmission des noms de personne au sein d'une communauté met en évidence les tendances «à la mode » dans l'acte de dénomination pour chaque période historique (la préférence pour un certain prénom ou pour des prénoms étrangers/ l'utilisation de noms traditionnels comme marque de l'identité, l'option pour des variantes graphiques différentes), ainsi que la manière dont ces tendances sont perçues par la société. Par exemple, les prénoms étrangers sont considérés par certains membres de la collectivité comme une déviation de la tradition, tandis que d'autres membres apprécient que ces noms répondent à une tendance du monde contemporain : l'innovation et la différenciation. Bien que les préférences anthroponymiques diffèrent d'un pays à l'autre, mais aussi à l'intérieur d'un pays ou d'une communauté à l'autre, elles sont réunies par une tendance commune : celle de satisfaire le goût du moment en matière de nom (voir aussi Troplini 2015 : 383). Pour une image de l'anthroponymie d'autres pays européens, dans le cadre de la migration, voir aussi Fernández Juncal (2018), Jordà \& Pujadas-Mora \& Cabré (2016) pour l'Espagne ; Eskola \& Hämäläinen (2019) pour la Finlande ; Arai et al. (2015), Grange (2016) pour la France ; Szabó T. (2018) pour la Hongrie ; Griķe (2019) pour la Lettonie ; Alhaug \& Saarelma (2017) pour la Norvège ; Troplini (2015) pour la Pologne; Aldrin (2009) pour la Suède ; et Abubakari (2020), Dechief (2009), Sabet \& Zhang (2020), Tonda \& Rossebastiano (2014), pour des communautés non européennes.

\subsection{Repères socio-onomastiques de la commune de Certeze, du dép. de Satu Mare}

L'anthroponymie de la région étudiée se caractérise par une grande créativité manifestée surtout par les prénoms féminins, plus accentuée que pour les prénoms masculins, aspect illustré dans le tableau ci-après présenté (Tableau 1). Le registre anthroponymique de Certeze que nous étudions 
s'inscrit dans la tendance de la modernisation des prénoms à la suite de l'émigration des Roumains et de l'influence des médias (voir aussi Felecan N. 2011, Semeniuc 2013, Tomescu 2017, Zăbavă 2013).

Tableau 1 : Statistique des prénoms de la commune de Certeze

\begin{tabular}{|c|c|c|c|c|c|c|}
\hline \multirow{3}{*}{ Fréquence } & \multicolumn{3}{|c|}{ Filles } & \multicolumn{3}{|c|}{ Garçons } \\
\hline & $\begin{array}{l}2000- \\
2010\end{array}$ & $\begin{array}{l}2011- \\
2020\end{array}$ & $\begin{array}{l}2000- \\
2020\end{array}$ & $\begin{array}{l}2000- \\
2010\end{array}$ & $\begin{array}{l}2011- \\
2020\end{array}$ & $\begin{array}{l}2000- \\
2020\end{array}$ \\
\hline & $\begin{array}{l}104 \\
\text { noms }\end{array}$ & $\begin{array}{l}113 \\
\text { noms }\end{array}$ & $\begin{array}{l}188 \\
\text { noms }\end{array}$ & $\begin{array}{l}80 \\
\text { noms }\end{array}$ & $\begin{array}{l}124 \\
\text { noms }\end{array}$ & $\begin{array}{l}163 \\
\text { noms }\end{array}$ \\
\hline & $\boldsymbol{\nabla}$ & $\boldsymbol{\nabla}$ & $\nabla$ & $\boldsymbol{\nabla}$ & $\nabla$ & $\nabla$ \\
\hline$\geq 20$ occurrences & 1 & 1 & 1 & 1 & - & 3 \\
\hline $\begin{array}{l}10-19 \\
\text { occurrences }\end{array}$ & - & - & 1 & 2 & 5 & 8 \\
\hline 5-9 occurrences & 1 & 6 & 8 & 6 & 10 & 11 \\
\hline 4-3 occurrences & 8 & 12 & 28 & 11 & 18 & 25 \\
\hline 2 occurrences & 14 & 29 & 39 & 12 & 18 & 24 \\
\hline 1 occurrence & 80 & 65 & 111 & 48 & 73 & 92 \\
\hline
\end{tabular}

Les 475 noms de personne, simples ou composés, analysés, comprennent dans leur structure 188 prénoms féminins, respectivement 163 prénoms masculins (en incluant les variantes graphiques des mêmes noms ou des correspondants d'autres langues, par exemple le roum. Darius - l'it. Dario, le roum. Ioan - le port. João, le roum. George - le fr. Georges - l'esp. Jorge, le roum. Robert - l'it. Roberto ; le roum. Iulia - l'it. Giulia). Sur les 104 noms féminins enregistrés pendant les années 2000-2010, 80 ont une seule occurrence et 14 ont deux occurrences. Après l'année 2000, le nombre des prénoms féminins qui apparaissent une seule fois dans les structures dénominatives analysées est réduit à 65 , le nombre des prénoms à la fréquence 2 augmentant à 29. Dans le cas des prénoms masculins, la situation est identique : pendant la première période, on a noté 12 noms à fréquence 2 et 48 avec une seule occurrence ; pendant la deuxième période, 18 noms à fréquence 2 , respectivement 73 avec une seule entrée. Ainsi, seulement 34 prénoms féminins, respectivement 40 masculins, restent constants dans toutes les deux périodes du temps (voir le Tableau 2). Les prénoms avec plus de cinq occurrences pendant 2000-2020 sont des hagionymes roumains ou des emprunts modernes considérés «à la mode », ayant une grande fréquence aussi dans d'autres régions du pays.

Tableau 2 : Fréquence des noms communs dans les deux périodes du temps analysées

\begin{tabular}{|c|c|c|}
\hline Fréquence & Filles & Garçons \\
\hline$\geq 20$ & 1 & 3 \\
\hline $10-19$ & 1 & 8 \\
\hline $5-9$ & 4 & 10 \\
\hline $4-3$ & 17 & 12 \\
\hline$=2$ & 12 & 7 \\
\hline
\end{tabular}


Dans le cas des prénoms masculins, les premières 7 positions, dans l'ordre décroissant de la fréquence pendant la période 2000-2020, avec plus de 13 mentions (voir le Tableau 3), sont occupées par des prénoms qui se rencontrent dans toutes les deux décennies soumises à l'analyse. Parmi les noms enregistrés avant et après 2010, ayant plus de 10 occurrences, on remarque : Alexandru, Daniel, Marian et avec entre 10 et 5 mentions :Damian, Dumitru, Patrick, Andrei, Gheorghe, Emanuel, Antonio, Sebastian, Ilie, Ștefan. En ce qui concerne les prénoms féminins, les 34 noms constants pendant les deux intervalles analysés occupent, dans le tableau général de la fréquence, pendant 2000-2020, des positions variables : Maria, Alexandra, les deux premières positions, avec plus de 10 mentions, Melissa, Antonia, Patricia, Georgiana, avec plus de 5 mentions.

Tableau 3 : Fréquence des noms ${ }^{8}$

\begin{tabular}{|c|c|c|c|c|c|c|}
\hline & 2000-2010 & 2011-2020 & $2000-2020$ & 2000-2010 & 2011-2020 & $2000-2020$ \\
\hline 1. & Vasile -20 & George-15 & Vasile -32 & Maria -23 & Maria -37 & Maria -60 \\
\hline 2. & Ionuţ-15 & Gabriel-13 & Ionuț-23 & $\begin{array}{l}\text { Alexandra- } \\
6\end{array}$ & $\begin{array}{l}\text { Alexandra } \\
-8\end{array}$ & $\begin{array}{l}\text { Alexandra } \\
-14\end{array}$ \\
\hline 3. & Ioan -10 & $\begin{array}{l}\text { Ioan, } \\
\text { Vasile- } 12\end{array}$ & Ioan -22 & $\begin{array}{l}\text { Adriana, } \\
\text { Claudia, } \\
\text { Melissa }-4\end{array}$ & Ioana -7 & $\begin{array}{l}\text { Ioana, } \\
\text { Melissa, } \\
\text { Sofia }-7\end{array}$ \\
\hline 4. & $\begin{array}{l}\text { Adrian } \\
\text { Denis - } 9\end{array}$ & David - 10 & $\begin{array}{l}\text { George - } \\
18\end{array}$ & $\begin{array}{l}\text { Andrea, } \\
\text { Camelia, } \\
\text { Denisa, } \\
\text { Georgiana, } \\
\text { Patricia - } 3\end{array}$ & $\begin{array}{l}\text { Sofia, } \\
\text { Maya-6 }\end{array}$ & $\begin{array}{l}\text { Antonia, } \\
\text { Maya, } \\
\text { Patricia- } \\
6\end{array}$ \\
\hline 5. & $\begin{array}{l}\text { Daniel } \\
\text { Emanuel-6 }\end{array}$ & Ionuț-8 & $\begin{array}{l}\text { Adrian - } \\
16 \\
\text { Gabriel - } \\
16\end{array}$ & & $\begin{array}{l}\text { Victoria- } \\
5 \\
\text { Antonia- } \\
5\end{array}$ & $\begin{array}{l}\text { Georgiana } \\
-5 \\
\text { Victoria- } \\
5 \\
\end{array}$ \\
\hline 6. & $\begin{array}{l}\text { Alexandru, } \\
\text { Dumitru }-5\end{array}$ & $\begin{array}{l}\text { Adrian, } \\
\text { Alexandru, } \\
\text { Damian-7 }\end{array}$ & David-14 & & $\begin{array}{l}\text { Karina }-4 \\
\text { Ana }-4\end{array}$ & $\begin{array}{l}\text { Adriana, } \\
\text { Alessia, } \\
\text { Ana, } \\
\text { Andrea, } \\
\text { Claudia, } \\
\text { Denisa, } \\
\text { Elisa, } \\
\text { Gabriela, } \\
\text { Karina, } \\
\text { Selena-4 }\end{array}$ \\
\hline 7. & $\begin{array}{l}\text { Antonio, } \\
\text { David, } \\
\text { Marian, } \\
\text { Sebastian-4 }\end{array}$ & $\begin{array}{l}\text { Andrei, } \\
\text { Daniel, } \\
\text { Kevin, } \\
\text { Marian, }\end{array}$ & Denis - 13 & & $\begin{array}{l}\text { Anna, } \\
\text { Carla, } \\
\text { Daria, } \\
\text { Elena, }\end{array}$ & $\begin{array}{l}\text { Amalia, } \\
\text { Anna, } \\
\text { Beatrice, } \\
\text { Camelia, }\end{array}$ \\
\hline
\end{tabular}

8 Les prénoms rencontrés dans toutes les deux périodes analysées sont écrits en gras. 


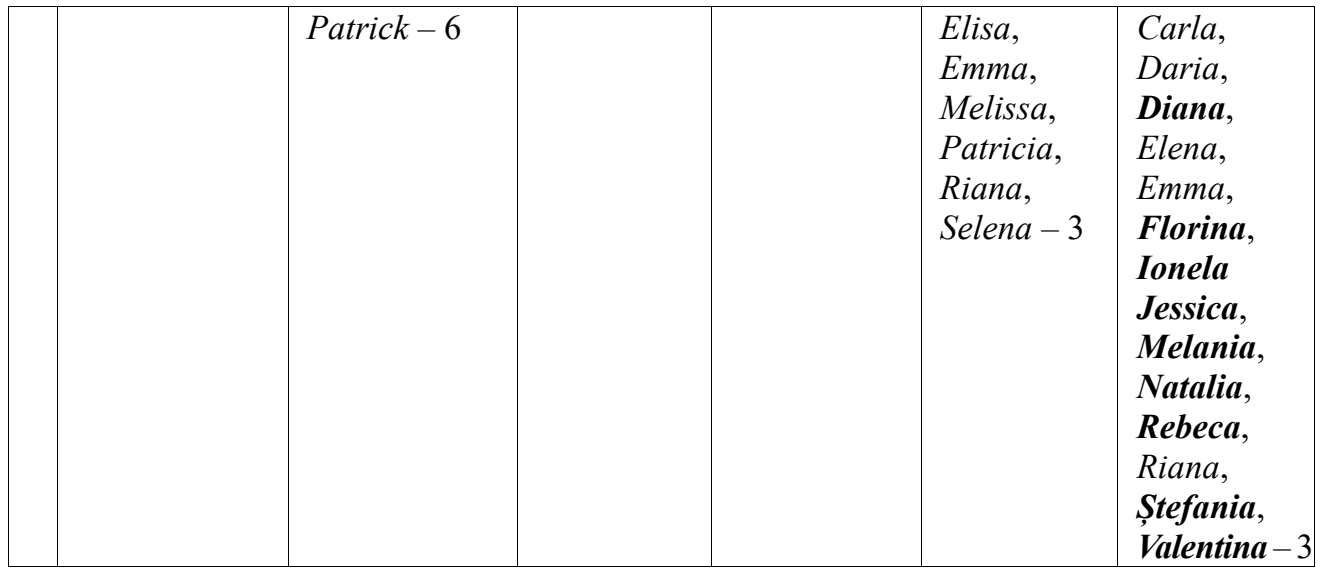

En perspective diachronique, le sous-système des prénoms masculins se caractérise par la stabilité de certains noms du calendrier religieux/ bibliques : Vasile, Ioan, Gabriel, David. Pourtant, la variante culte George remplace le traditionnel Gheorghe, et le diminutif Ionuț est préféré au roumain Ioan. David, bien qu'il soit rencontré aussi avant 2010, est considéré un nom «à la mode » après 2015, tandis que Denis, occupant la $7^{\mathrm{e}}$ place ces derniers temps, disparaît petit à petit du système onomastique des habitants de Certeze après 2010.

En ce qui concerne les prénoms féminins, on constate une grande variété, à partir des emprunts classiques jusqu'aux variantes occidentales. Malgré cela, on peut remarquer la préférence des parents pour des noms considérés paneuropéens comme Maria ou Alexandra, ainsi que des tendances anthroponymiques rencontrées dans des aires étendues : par exemple, le remplacement du nom Denisa, après 2010, avec Sofia ou Maya. Ce qui nous semble intéressant est l'absence de certains noms du calendrier religieux roumain, connus pour les grands-parents et les parents, qui ont été remplacés, en première instance, avec des diminutifs/ hypocoristiques, ensuite avec leurs correspondants des langues occidentales. Par exemple, les prénoms roumains Mihail et Nicolae sont remplacés, pendant la période analysée, par le fr. Michel (rencontré dans les deux intervalles de temps discutés) ou par l'angl. Mickael ${ }^{9}$ (après 2010), respectivement par l'hypocoristique roumain Nicu ou le diminutif Nicuşor (2000-2010) et le fr. Nicolas (après 2010). Le prénom roumain Ileana n'entre pas dans la série des prénoms discutés, bien qu'on puisse rencontrer des porteurs de ce nom parmi les parents des enfants enregistrés pendant la période soumise à l'analyse. Mais après 2010, on a noté la forme cultivée Elena. Cet aspect peut être expliqué, dans le cas des enfants nés à l'extérieur du pays, par le désir des parents de choisir un nom nouveau, proche du pays où ils ont émigré, mais gardant en quelque sorte le principe dénominatif selon un membre de la famille et pour les enfants nés en Roumanie, par imitation et par désir de différenciation.

9 La forme anglaise du nom est Michael; Mickael est l'une des variantes plus récentes. 
Pendant la période 2000-2010, sont préférés des prénoms qui représentent soit des emprunts occidentaux récents, soit classiques et d'autres sont enregistrés à Certeze après l'année 2011. Par exemple, dans l'intervalle 2000-2010, on retrouve :

- les prénoms masculins : Alessandro (2) ${ }^{10}$, Alexandre (3), Carlos (1), Călin (1), Cătălin (2), Ciprian (1), Felician (1), Giorgi (2), Hakime (1), Jorge (1), Julian (2), Lucian (1), Marco (1), Mădălin (1), Raul (1), Roberto (1), Ruben (1), Serge (1), Sergiu (1);

- les prénoms féminins: Adriana (4), Alessandra (1), Alicia (1), Antonella (1), Camelia (3), Carmen (1), Carolina (2), Celina (1), Claudia (4), Emanuela (1), Erika (2), Katalina (1), Katerina (1), Larissa (1), Laura (1), Loriana (1), Maylise (1), Mihaela (1), Milena (1), Mirabela (1), Paula (1), Rihanna (1), Romina (1), Sonia (2), Soriana (1), Sorina (1), Tania (1), Tonia (1), Vanesa (2), Veronica (1). Certains d'entre eux sont abandonnés après 2010 ou remplacés par d'autres variantes graphiques ou par des formes empruntées d'autres langues : Eric est remplacé par Erik, Roberto apparait avec la variante roumaine Robert, avant et après 2000 ; Mélanie n'apparait après l'année 2010, mais on rencontre les formes Melany et Melania, Rihanna apparait avec la forme graphique Riana, Vanesa est remplacé par la variante Vanessa. De même, après 2010 on rencontre une série de noms nouveaux : Alin (1), Ansael (1), Aurelian (1), Ayan (3), Cosmin (1), Dario (2), Elias (2)/ Elyas (4), Emil (1), Emilian (1), Kevin (6), Laurențiu (1), Luca (3), Manuel (1), Maximilian (2), Maxyme (1), Rayan (4), Ricardo (1), Vladimir (1)// Alexia (1), Andra (2), Bianca (2), Casandra (1)/ Cassandra (2), Cataleya (1), Clara (2), Crina (1), Daiana (2), Dalia (1), Daria (3), Davya (1), Felicia (1), Giorgiana (1), Lavinia (2), Miruna (1), Nell (1), Olivia (1), Sofia (7), Victoria (5), Zoe (1).

Quant à l'origine des prénoms analysés, on remarque un accroissement progressif des noms étrangers (Figure 2), qui sont des emprunts récents aux langues occidentales, adaptés ou non à la langue roumaine ou ayant la graphie selon les principes occidentaux, à côté de noms hébreux, d'origine biblique, « ressuscités » par l'intermédiaire des cultes religieux néo-protestants et transmis par imitation. Un grand nombre de prénoms est représenté par les emprunts classiques du latin ou par les prénoms étrangers, empruntés auparavant de langues occidentales, également utilisés de façon relativement fréquente avant l'année 2000, suivis par les diminutifs et les hypocoristiques. Ce qui est intéressant est le fait que, bien que variés, les emprunts occidentaux, à des rares exceptions, surtout dans le cas des prénoms féminins, entrent dans la catégorie des noms à fréquence 3 ou inférieure.

10 Le chiffre entre parenthèses indique la fréquence dans la période mentionnée. 


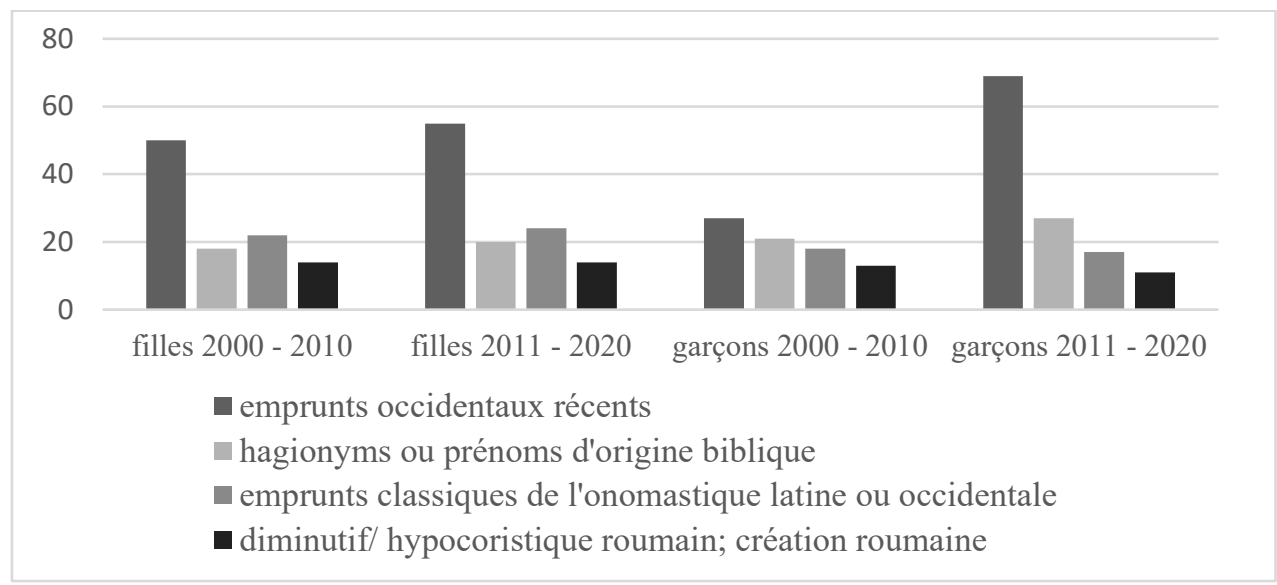

Figure 2 : Origine des noms

L'emprunt de prénoms d'une autre langue peut exprimer également le fait que le groupe social respectif se trouve dans un processus de changement de l'identité culturelle, aspect signalé surtout dans le cas des migrants, qui essaient, par l'intermédiaire de l'onomastique, de s'identifier du point de vue culturel à l'état d'adoption. La propagation de ces noms dans les familles roumaines s'est réalisée d'une part par imitation, comme conséquence du désir des parents de chercher un nom nouveau, et d'autre part, à la suite de l'influence exercée par la culture du pays de résidence et par les médias. Ainsi, certains noms étrangers sont-ils dus à la préférence des parents pour des personnalités du football (Cristiano Ronaldo), de la musique (Rianal Rihana), des personnages de film (Sabrina), etc. Le prénom Chanelia est choisi d'après le nom de la célèbre couturière Coco Chanel. Quant au nom des enfants nés en Roumanie, qui habitent le pays et y fréquentent l'école, on remarque un degré conservatoire plus élevé.

En ce qui concerne le renouvellement du registre anthroponymique, on peut constater que, ces dernières décennies, les enfants de Certeze ont reçu des noms ${ }^{11}$ :

- italiens : Alessio, Amalio, Antonio, Cristiano, Enzo, Giorgio, Giorgi, Giuseppe, Lorenzo, Marco, Mario, Matteo (écrit, aussi, Matheo, Mattéo), Pedro, Riccardo, Roberto, Simone, Timeo // Alessia, Antonia, Antonella, Ariana, Elia, Federica, Florena, Francesca, Giorgia, Giulia, Isabella, Melissa, Patrizia, Raffaela ;

- français : Alexandre, Denis, Hugo, Michel, Nicolas, Yan // Celine, Mélanie, Nicole, Pascaline;

- anglais : Kevin, Mickael, Patrick (écrit, aussi, Patric, Patryck) // Emily, Emma, Jennifer (écrit, aussi, Jenifer, Jennyfer), Mary;

- hispaniques : Carlos, Fernando, Jorge, Maria // Amanda, Damaris, Iris ;

- germaniques : Albert, Armin // Adela, Adelina, Erika, Karla, Maylise;

11 Nous donnons l'origine des noms après Ionescu (2001). 
- slaves : Andrzej, Milann, Serge // Bojana, Ivanovna;

- orientaux : Ali, Ayan, Mahmet, Samir // Rania.

Il faut noter que, quoique la plupart des migrants soient établis en France, les prénoms français occupent la deuxième place, après les prénoms italiens, mais ils sont suivis par les prénoms anglais. Cet aspect peut être expliqué par les tendances anthroponymiques « à la mode " tant au pays qu'à la diaspora, par la préférence pour les noms italiens, faciles à prononcer, fréquents parmi les migrants en général, mais aussi parmi les non migrants. En ce qui concerne les noms anglais, ils deviennent " une mode » au pays, mais aussi dans les communautés roumaines d'autres états européens, par exemple d'Italie (voir aussi Mihali 2021).

Une constante dans les choix anthroponymiques des migrants, mais aussi des non migrants est représentée par les noms religieux, aspect valable pour tout le territoire roumain (voir aussi Tomescu 2017 : 305, Felecan N. 2011 : 50). Les noms bibliques, surtout ceux d'origine hébraïque, actualisés par l'intermédiaire des cultes néo-protestants, ont été repris par les parents appartenant à d'autres confessions, parce qu'ils ont été perçus comme modernes et innovants. Certains noms ont été enregistrés seulement pendant la période 2000-2010, par exemple Samuel ; Debora, Naomi, d'autre noms sont entrés dans le système onomastique de Certeze après 2010, comme une conséquence naturelle des tendances onomastiques de Roumanie : Luca; Abigail, Meriam, Rahela, Sara.

La grande fréquence des hagionymes dans l'anthroponymie de Certeze s'explique par le respect d'un ancien principe dénominatif : la transmission d'un prénom d'une génération à l'autre. Par conséquent, les enfants sont baptisés selon leur parents, leur grands-parents, les parrains ou d'autres proches de la famille : le père Ioan - la fille Ioana Karina ou, dans un autre cas, le fils Ioan, le père Dumitru - le fils Dumitru, le père Gheorghe - le fils Gheorghe, le père Petrică - le fils Petrică Ionel, le père Vasile - le fils Adrian Vasile, la mère Daniela - le fils Daniel, la mère Maria - la fille Maria Elisa. Parfois, les noms du calendrier religieux, noms des parents, sont remplacés par des variantes cultivées (le père Ghiță, l'hypocoristique de Gheorghe - le fils Damian George, le père Gheorghe - le fils George Marian), par des formes considérées paneuropéennes (la mère Marioara - la fille Melisa Maria), ou par des dérivés/ diminutifs (le père Gheorghe - la fille Florica Georgiana, le père Petrică - la fille Petruța). Dans les familles formées de plusieurs membres, un enfant prend souvent le nom du père, l'autre, le nom de la mère (le père Dumitru, la mère Tania - le fils Dumitru Adrian, la fille Tania Adriana). Dans certains cas, le prénom qu'on veut garder en famille est remplacé avec un correspondent d'une autre langue : la mère Maria - le fils Mario, le père Petru - le fils Patrick Jr. (ici le sens du nom anglais Patrick est confondu avec le sens du nom roumain Petru). On peut donc constater que, par le nom, on fait une différenciation entre les générations, à l'intérieur de la même famille, par des diminutifs, des hypocoristiques et des variantes cultivées et à l'intérieur d'une communauté, par des préférences 
anthroponymiques différentes (voir aussi Troplini 2015, Fernández Juncal 2018).

Le désir de garder un nom en famille, associé au besoin d'individualisation et de différenciation, mais aussi à la tendance d'emprunter des noms nouveaux, innovantes, en français ont conduit à l'utilisation des diminutifs et des hypocoristiques comme prénoms proprement dit et plus stables. Il s'agit des hypocoristiques tels que : Alex, Cristi, Mitică, Nicu, Sebi, Theo // Anka, Mia, Oana, Sandra et des diminutifs : Dănuț, Florinel, Georgel, Ionuț, Ionel, Mitruț, Nicuşor, Petrișor, Romică, Sorinel // Ancuța, Anişoara, Dumitrița, Florica, Petruța. On ne peut pas ignorer le fait que les hypocoristiques et les diminutifs masculins sont majoritaires, aspect explicable en partie par la grande variété du registre anthroponymique féminin, les prénoms masculins à fréquence élevée étant peu nombreux, d'où le besoin d'originalité.

Un autre trait du système anthroponymique de Certeze, dû à la migration, mais aussi à la globalisation, est l'utilisation d'un prénom dans sa forme roumaine à côté de son correspondent des autres langues. Le prénom roumain Alexandru, orthographié aussi Alesandru, nom qui reste constant dans les deux périodes analysées, circule en parallèle avec le sl. Aleksandru, l'it. Alessandro, le fr. Alexandre et à côté de l'hypocoristique paneuropéen Alex. D'autres exemples sont: Christian, avec la variante orthographique Cristian, - l'it. Cristiano et l'hypocoristique Cristi, le roum. Damian, avec la variante orthographique Damyan - le fr. Damien, le roum. Eduard - l'it. Edoardo - le fr. Edouard l'angl. Edward, le roum. Grigore, Grigorie - l'angl. Gregory, le roum. Mateil'it. Matheo, Mattéo, Matteo - le magh. Mattia, le roum. Petru, avec la variante Petre - l'angl. Patrick, avec les variantes Patryck, Patric, - l'esp. Pedro.

Pour les prénoms féminins, la situation est différente. À l'exception de quelques prénoms (le roum. Alexandra - le sl. Aleksandra, l'it. Alessandra, Carolina - le fr. Caroline (écrit, aussi, Karoline), le roum. Iulia - l'it. Giulia l'esp. Julia, le roum. Melania - le fr. Mélanie - l'angl. Melany) qui circulent à côté de leur correspondant dans les autres langues, il n'y a pas de traductions des noms, mais leurs variantes avec des orthographies différentes sont fréquentes, les unes dues à l'intention de garder la graphie de la langue d'origine, mais sans connaître parfois la manière correcte d'écrire le prénom respectif, les autres, dues au besoin d'adaptation des noms aux normes de la langue roumaine.

Dans certaines situations, en particulier dans le cas de la graphie avec $-y$-, on peut parler de l'intention des parents de mettre en évidence l'enfant par son nom. Il s'agit donc de prénoms tels : Ana - Anna, Anamaria - Anamareia Annamaria, Ariana - Arianna, Carla - Karla, Casandra - Cassandra, Christina - Cristina, Ioana - Joana, Marina - Marynna, Melisa - Melissa, Patricia - Patrisia - Patrizia, Riana - Rihanna, Teodora - Theodora, Vanesa Vanessa, Iasmina - Yasmina ; Casian - Cassian, Ianis - Yanis, Yannis, MarianMaryan, Rafael - Raphael, Rafaiel. On remarque aussi le cas intéressant de deux formes : Yoan, forme rencontrée deux fois entre les années 2000 et 2020, et Yonut, forme ayant une seule occurrence avant 2010 ; ce sont des variantes 
graphiques avec $y$ de deux prénoms masculins roumains : Ioan, respectivement Ionut, les deux avec une grande fréquence. En grande partie, ces variantes graphiques sont étroitement liées au niveau d'éducation des parents (voir le roum. Andreia, Andrea pour Andreea; Rafaiel pour Rafael), mais aussi au niveau de la langue du «pays d'accueil» du migrant/ de l'immigrant est « bas », «peu élevé. Très souvent, les parents, lorsqu'on leur demande pourquoi ils ont écrit le nom de leurs enfants comme ça, répondent : « on écrit comme ça en français/ en italien/ en allemand, etc. ", mais la variante qu'ils proposent diffère de la variante originale (par ex. Maxyme < le fr. Maxime ; Ricardo < l'it. Riccardo ; Melanie < le fr. Mélanie). Nous mentionnons également le prénom Braian (écrit aussi Brayan) et Raian (écrit aussi Rayan), orthographiés selon le principe phonétique, même si les parents pensaient à un prénom anglais, Brian, respectivement Ryan. Le doublement de certaines consonnes ou la graphie avec $y, k$, là où ces lettres ne s'expliquent pas par l'origine du nom (Anka, Yonuţ), parce qu'elles ne sont pas étymologiques, représentent des déviations et des défis de la norme, une séparation du traditionnel considéré commun, dans le but d'épater et d'être différent.

Un autre élément de modernité est représenté par la structure des prénoms. Si les parents sont baptisés avec un seul ou, plus rarement, deux prénoms, les enfants portent surtout des noms doubles ou triples. Des 475 noms analysés, 408 (86\%) sont composés (341 doubles, 66 triples, 1 quadruple : Alexandre Denis Ioan $\mathrm{Nicu})$ et 67 (14\%) sont simples. Les combinaisons sont variées et inédites :

- nom d'origine biblique ou hagionyme en roumain + emprunt récent : Anais Maria, Giorgia Naomi, Jessica Maria, Maria Adelina, Sara Georgeona // Alessandro Marian Vasile, Denis Yoan, Fernando Vasile, Gabriel Alexandre Vasile, Luis Ilie, Kevin Vasile, Kevina Ioan George, Milann Gabriel, Roberto Ioan, Ruben Ioan, Sebastien Vasile George, Theo Vasile Michel, Vasile Patrick;

- emprunts récents : Antonella Jennyfer, Caroline Celine, Francesca Raffaella, Giorgia Alessandra, Patricia Denisa Florena // Albert Ilyan, Alessio Nicolas, Edward Patrick, Jorge Maria, Matteo Ayan Patrick, Mickael Hugo, Rayan Yannis ;

- prénom/ diminutif/ hypocoristique roumain + emprunt moderne : Alexandra Francisca, Dumitrița Patricia, Filipa Alexandra, Jenifer Claudia, Selena Crina Pascaline // Dario Florin, Dorian Gregory Florian, Enzo Adrian, Ethan Adrian, Laurențiu Yannis Yonuț, Mael Adrian, Mario Maximilian, Patrick Fabian Aurelian, Sebastien Ionuț ;

- prénoms roumains : Alexandra Maria, Daniela Maria, Eva Mariana, Mihaela Maria // Daniel David, Daniel Vasile, David George Ionel, Ionuț Daniel Marian, Ionuț Mădălin, Romică Daniel, Sebi Dănuț.

On peut remarquer le fait que la plupart des prénoms composés contiennent un nom du calendrier (ou un diminutif provenu d'un hagionyme 
roumain), sur des positions différentes à l'intérieur de la structure dénominative, à côté d'emprunts de la même langue ou de langues différentes : Elias Petre, Ionuț Marco Antonio, Serge Denis Ștefan, Antonio Andrei Patrick, Yann Andrzej Dumitru // Riana Alessia Maria, Selena Maria Sofia. Étant donné que la plupart des noms bilingues/ trilingues sont attribués aux enfants provenant des familles de migrants (ou qui sont nés pendant la période dans laquelle leurs parents ont travaillé à l'étranger), l'emploi dans la même structure d'un nom étranger à côté d'un hagionyme roumain peut être expliqué par le double statut des migrants : résidents d'un pays étranger, voulant s'y intégrer, et roumains par le sang, par la culture et par tradition. Les noms étrangers reflètent donc le désir d'intégration, de changement culturel, tandis que les noms traditionnels roumains indiquent l'essai de protéger l'identité culturelle (Sabet \& Zhang $2020: 3$ ) d'origine.

Dans le cas des familles mixtes, les noms des enfants sont choisis de telle manière qu'ils plaisent aux deux parents (pour l'acte de prénommer, dans les couples mixtes en France, voir Collet 2019). Ainsi, on rencontre des situations dans lesquelles les enfants sont nommés avec des noms spécifiques pour les deux cultures. Par exemple, l'un des deux enfants de la famille mixte roumaine-portugaise Pop Afonso Ribeiro (nom de famille roumain Pop + nom portugais Afonso Ribeiro) porte un nom double, Filipa Alexandra: un prénom roumain, rarement utilisé aujourd'hui, Filipa, probablement, sur l'influence de la variante portugaise, Filipe, et un prénom roumain, et l'autre enfant - deux prénoms portugais, João Pedro. La même situation est rencontrée aussi dans le cas de l'enfant Turkmet Mehmet Ali Cătălin, nommé avec deux prénoms spécifiques de la culture arabe et un prénom roumain. Parfois, à l'intérieur des familles mixtes, les parents choisissent des noms modernes, paneuropéens, sans renvoyer à une certaine culture (Aiteur Emma Anabella Meriam).

De même, une partie des noms slaves apparaissent dans les familles composées des ethnies slovaque et ukrainienne, fait soutenu aussi par le nom de famille : Idvorjan Aleksandra, Idvorjan Aleksandru (frères), Iovdi Maria Ivanovna, Marjanović Bojana.

La contradiction entre le nom de famille et la forme du prénom, à la suite de l'emprunt massif de noms occidentaux, « inadaptés » à la langue roumaine, renforce l'idée que, à l'époque de la globalisation, les anthroponymes ne sont plus pertinents pour l'ethnie du porteur, ni pour l'identification d'une tradition onomastique. On arrive donc à des situations inédites telles que : Codreanu Ethan Adrian, Creștin Amalio Alin, Olariu Anais Melissa Bernadette, Pop Yasmina Damaris, Pricop Hakime.

\section{Conclusions}

L'analyse des données recueillies met en évidence une évolution de l'anthroponymie traditionnelle roumaine, basée en grande partie sur des hagionymes et sur des emprunts classiques du latin ou des créations roumaines, 
à un nouveau répertoire onomastique, d'une certaine façon, étrangers aux normes sociales et traditionnelles des habitants de Certeze, mais devenu un stéréotype pour les Roumains, soit de l'étranger, soit du pays.

Les deux dernières décennies, caractérisées par l'accroissement de la migration, ont conduit aux changements anthroponymiques majeurs, étroitement liés aux événements sociopolitiques, aux mouvements de la population, à la perception de l'altérité, à la construction de l'identité, à la projection d'un statut social par le nom pour l'enfant, etc. D'une perspective diachronique, on remarque un certain schéma anthroponymique, spécifique pour chaque étape de temps, ainsi qu'une dynamique des noms de personne qui disparaissent facilement, mais qui peuvent également être empruntés et adaptés aussi rapidement. En conclusion, le système anthroponymique de Certeze offre une image de l'acculturation linguistique, l'onomastique représentant un témoin et un instrument pour l'analyse de l'évolution historique des mentalités.

\section{Bibliographie}

Abubakari, Hasiyatu. 2020. Personal names in Kusaal: A sociolinguistic analysis. Language \& Communication 75, 21-35.

Aldrin, Emilia. 2009. The choice of first names as a social resource and act of identity among multilingual families in contemporary Sweden. Dans Ahrens, Wolfgang \& Embleton, Sheila \& Lapierre, André (eds.), Names in multi-lingual, multi-cultural and multi-ethnic contact. Proceedings of the 23rd International Congress of Onomastic Sciences. Toronto, 17-22 August 2008, 86-92. Toronto : York University. (https://yorkspace.library. yorku.ca/xmlui/handle/10315/2926) (Page consultée en mai 2019.)

Aldrin, Emilia. 2014. Choosing a name $=$ Choosing identity? Towards a theoretical framework. Dans Tort i Donada, Joan \& Montagut i Montagut, Montserrat (eds.), Names in daily life. Proceedings of the XXIV International Congress of Onomastic Sciences, 392-401. Barcelona : Generalitat de Catalunya. (http://www.gencat.cat/llengua/BTPL/ICOS2011/044.pdf) (Page consultée en mai 2019.)

Aldrin, Emilia. 2017. Creating identities through the choice of first names. Dans Ainiala, T. \& Östman, J. (eds.), Socio-onomastics: The pragmatics of names, 45-68. Amsterdam: John Benjamins.

Alhaug, Gulbrand \& Saarelma, Minna. 2017. Naming of children in Finnish and Finnish-Norwegian families in Norway. Dans Ainiala, Terhi \& Östman, Jan-Ola (eds.), Socio-onomastics: The pragmatics of names, 70-91. Amsterdam: John Benjamins.

Arai, Mahmood \& Besancenot, Damien \& Huynh, Kim \& Skalli, Ali. 2015. Children's first names, religiosity and immigration background in France. International Migration 3(6). (http://halshs.archives-ouvertes.fr/halshs00383090/fr/) (Page consultée en mars 2021.) 
Bucholtz, Mary. 2003. Sociolinguistic nostalgia and the authentication of identity. Journal of Sociolinguistics 7(3), 394-416.

Collet, Beate. 2019. Prénommer son enfant dans les couples mixtes. Stratégies d'ajustements interculturels et logiques de genre. Recherches familiales 1(16), 155-167. (https://www.cairn.info/revue-recherches-familiales2019-1-page-155.htm) (Page consultée en mars 2021.)

Dechief, Dianne. 2009. Forms and norms: Theorizing immigration-influenced name changes in Canada. Dans Ahrens, Wolfgang \& Embleton, Sheila \& Lapierre, André (eds.), Names in multi-lingual, multi-cultural and multiethnic contact. Proceedings of the $23 \mathrm{rd}$ International Congress of Onomastic Sciences. Toronto, 17-22 August 2008, 267-275. Toronto: York University. (https://yorkspace.library.yorku.ca/xmlui/handle/10315/3645) (Page consultée en mai 2019.)

Diminescu, Diana. 1996. Deplasările oșenilor în străinătate, un nou model de migrație [Les mouvements des habitants d'Oaș à l'étranger, un nouveau modèle de migration]. Revista de cercetări sociale 2. (https://adatbank. transindex.ro/html/cim_pdf421.pdf) (Page consultée en mars 2021.)

Eskola, Ksenia \& Hämäläinen, Lasse. 2019. Given names of Russian-speaking children born in Finland between 2000-2018. Onoma 54, 197-217. (https://onomajournal.org/wp-content/uploads/2020/06/Onoma-54-1.10Eskola-H\%C3\%A4m\%C3\%A41\%C3\%A4inen-final-1.pdf) (Page consultée en mars 2021.)

Felecan, Nicolae. 2011. Dinamica numelor de botez reflectată în antroponimia municipiului Baia Mare [La dynamique des noms de baptême reflétée dans l'anthroponymie de Baia Mare]. Dans Oliviu Felecan (ed.), Proceedings of the First International Conference on Onomastics "Name and Naming": Multi-Ethnic Connections in Anthroponymy, Baia Mare, September 1921, 2011, 49-56. Cluj-Napoca : Editura Mega.

Felecan, Oliviu. 2013. Un excurs onomastic în spațiul public românesc actual [Une excursion onomastique dans l'espace public roumain actuel]. ClujNapoca : Mega, Argonaut.

Fernández Juncal, Carmen. 2018. Evolution of anthroponyms in an area of linguistic transition : A socio-onomastic study. Names 66(2), 85-95. (https://ans-names.pitt.edu/ans/article/view/2153/2152) (Page consultée en novembre 2020.)

Grange, Cyril. 2016. Nommer : enjeux symboliques, sociaux et politiques. Annales de démographie historique 1(131), 5-11. (https://www.cairn.info/revueannales-de-demographie-historique-2016-1-page-5.htm) (Page consultée en mars 2021.)

Griķe, Anna Elizabete. 2019. Sociocultural inscriptions in a personal name : A case study of name-giving in Latvia. Onoma 54, 181-196. (https:// onomajournal.org/wp-content/uploads/2020/06/Onoma-54-1.09-Grikefinal.pdf) (Page consultée en mars 2021.) 
Ionescu, Christian. 2001. Dicționar de onomastică [Dictionnaire d'onomastique]. N.p. : Elion.

Jordà, Joan Pau \& Pujadas-Mora, Joana Maria \& Cabré Anna. 2016. Surnames and migrations : The Barcelona area (1451-1900). Dans Hough, Carol \& Izdebska, Daria (eds.), 'Names and their environment'. Proceedings of the 25th International Congress of Onomastic Sciences, Glasgow, 25-29 August 2014, Volume 3 : Anthroponomastics, 131-143. Glasgow: University of Glasgow. (https://www.gla.ac.uk/media/media_576597_en.pdf) (Page consultée en mai 2019.)

Mihali, Adelina Emilia. 2021. Influences italiennes dans l'anthroponymie de la localité de Borșa (dép. de Maramureș, Roumanie). Dans Schøsler, Lene \& Härmä, Juhani (éds.) avec la collaboration de Lindschouw, Jan, Actes du XXIXe Congrès international de linguistique et de philologie romanes (Copenhague, 1-6 juillet 2019), Volume 1, 669-680. Strasbourg : Société de Linguistique Romane / Éditions de linguistique et de philologie.

Sabet, Peyman \& Zhang, Grace. 2020. First names in social and ethnic contexts: A socio-onomastic approach. Language \& Communication 70, 1-12.

Semeniuc, Sorin Cristian. 2013. Pragmatism în numirea copiilor, pe fondul migrației [Pragmatisme dans la dénomination des enfants, dans un contexte de migration]. Dans Ichim, Ofelia \& Botoșineanu, Luminița \& Butnaru, Daniela \& Clim, Marius-Radu \& Olariu, Florin-Teodor \& Tamba, Elena (eds.), Cultură și identitate românească - Tendințe actuale și reflectarea lor în diaspora. Iași : Editura Universității „Alexandru Ioan Cuza”. (http://www.philippide.ro/cultura_2010/ 34SEMENIUC\%20Sorin\%20final.pdf) (Page consultée en mai 2019.)

Suciu, Coriolan. 1967. Dicționar istoric al localităților din Transilvania [Dictionnaire historique des localités de Transylvanie], vol. I. [București] : Editura Republicii Socialiste România.

Szabó T., Annamária Ulla. 2018. Bilingualism: binominalism? Onomastica Uralica 11, 17-30. (https://edit.elte.hu/xmlui/bitstream/handle/10831/ 43635/T.\%20Szab\%C3\%B3\%20A_Bilingualism.pdf?sequence=1\&am p;isAllowed=y) (Page consultée en mai 2019.)

Tomescu, Domnița. 2017. Dinamica denominației personale primare în antroponimia actuală [La dynamique de la dénomination personnelle primaire dans l'anthroponymie actuelle]. Dans Topală, Dragoș Vlad (ed.), Cercetări lingvistice. Omagiu doamnei profesoare Adriana Stoichițoiu Ichim, 292-309. Craiova : Editura Sitech.

Tonda, Alfredo Juan \& Rossebastiano, Alda. 2014. La onomástica de los emigrados italianos en Argentina. Dans Tort i Donada, Joan \& Montagut i Montagut, Montserrat (eds), Names in daily life. Proceedings of the XXIV International Congress of Onomastic Sciences, 642-653. Barcelona : Generalitat de Catalunya. (http://www.gencat.cat/llengua/BTPL/ ICOS2011/068.pdf) (Page consultée en mai 2019.) 
Troplini (Abdurahmani), Edlira. 2015. Anthroponyms as (conventional or unconventional) parts in the development of language. Dans Felecan, Oliviu (ed.), Proceedings of the Third International Conference on Onomastics "Name and Naming" Conventional/Unconventional in Onomastics, Baia Mare, September 1-3, 2015, 376-385. Cluj-Napoca: Mega, Argonaut.

Watzlawik, Meike \& Guimarães, Danilo Silva \& Han, Min \& Jung, Ae Ja. 2016. First names as signs of personal identity: An intercultural comparison. Psychology \& Society 8(1), 1-21.

Zăbavă, Camelia. 2013. Tendințe noi în antroponimia din diaspora [Nouvelles tendances de l'anthroponymie de la diaspora]. Dans Ichim, Ofelia \& Botoșineanu, Luminița \& Butnaru, Daniela \& Clim, Marius-Radu \& Olariu, Florin-Teodor \& Tamba, Elena (eds.), Cultură și identitate românească - Tendințe actuale și reflectarea lor în diaspora. Iași : Editura Universității „Alexandru Ioan Cuza”. (http://www.philippide.ro/ cultura_2010/68ZABAVA\%20Camelia\%20final.pdf) (Page consultée en mai 2019.) 